\title{
Selbstdisziplin - Der Schlüssel
}

\author{
C. Thomas, R. Barta
}

Bitzer-Professur für Kälte-, Kryo- und Kompressorentechnik, Institut für Energietechnik, Fakultät Maschinenwesen, TU Dresden

\begin{abstract}
Die Covid19-Pandemie zeigt in allen Ebenen nur allzu deutlich die Stärken aber auch die jeweiligen Schwächen des Bildungssystems auf. Von geschlossenen Kindertageseinrichtungen, Grundschulen, weiterführenden Schulen, Fachhochschulen bis hin zur universitären Ausbildung sind die bildungstechnischen, persönlichen und sozialen Folgen des ausgesetzten Unterrichts bzw. der digitalen Varianten nicht absehbar. In allen Bildungsbereichen schien man nahezu unvorbereitet mit dem digitalen Zeitalter konfrontiert worden zu sein. Das Online-Format als solches schien bisher nicht in das (deutsche) Bildungssystem zu passen. Die Frage nach dem warum stellt sich hier nur allzu offensichtlich, da sowohl Schüler nahezu jeden Alters als auch Studierende täglich privat online sind. Das vorliegende Paper stellt kritische Fragen, wie online-Lehre sinnvoll für Lehrkräfte und Schüler/ Studierende umgesetzt werden kann, wo der Grundstock eines zielführenden online-Unterrichts gelegt werden muss und bei wem welche Zuständigkeiten für eine produktive Ausbildung liegen. Das umfangreiche Angebot an vertonten und visualisierten Kursen ist nur eine Seite der Medaille. Auf der anderen Seite stehen Themen wie Selbstdisziplin und -organisation. Sowohl die kindliche Ausbildung (Grundschule) als auch die Ausbildung an der Universität werden im Rahmen dieser Veröffentlichung aus Sicht der Autoren diskutiert und Kommentare sowie entsprechende Vorschläge zur Optimierung der Ausbildung auf Seiten der Lehrenden und Lernenden unterbreitet.
\end{abstract}

The Covid19 pandemic clearly showed the strengths and weaknesses of educations systems at all levels. From closed kindergartens, gradeschools and highschools to universities, the educational, personal and social impacts of the suspended education system are inconceivable. In all areas of education, it was shown that the digital age was nearly completely unprepared to confront these challenges. To date the online format has proven to not fit to the (German) education system. The question that arises is why is this the case, since schoolchildren of almost every age are online privately every day. The following paper poses critical questions such as how online education can be applied in a way that is reasonable for both students and teachers, where the foundation of a purposeful online lecture must be laid and by who do which portions of responsibility for a productive education lay? The extensive offer of recorded and visualized courses is only one side of the coin. On the other side lay topics such as self-discipline and organization. Both the gradeschool and university education are discussed by the authors in this publication, as are corresponding suggestions for optimization of the education from the sides of the teachers and students.

*Corresponding author: christiane.thomas@tu-dresden.de 


\section{Einleitung}

Im Rahmen der Vorbereitung der Lessons Learned-Veranstaltungen im Herbst 2020 sowie im Frühjahr 2021 fanden eine Vielzahl an Diskussionen zwischen den Autoren dieser Veröffentlichung statt, warum sich die aktive Teilnahme am Lehr-Lern-Prozess so ruckartig ändert, wenn ein Wechsel von Präsenz- hin zur Online-Lehre stattfindet, ob es in den Bildungssystemen der USA und Deutschlands ähnliche Phänomene gibt und welche Rückschlüsse man für die Zukunft daraus ziehen sollte.

Primäre Fragen sind: Wie lernen wir? Wie fand das Lernen und Lehren früher statt, wie heute und wie sollte der Lernprozess zukünftig begleitet werden?

Die mit der Covid19-Pandemie einhergehenden privaten Herausforderungen spielen selbstverständlich auf der Seite der Lehrer und Dozenten sowie bei den Schülern und Studierenden eine immense Rolle in der Gestaltung der Lehre, des Lernens und auch in der zeitlichen Planung. Auf diesen Bereich soll allerdings im Folgenden nicht tiefer eingegangen werden, da sich hierbei ein zu diverses Bild ergibt, was nicht zwangsläufig zu generellen Aussagen für die zukünftige Lehre führt.

Im Rahmen dieser Veröffentlichung soll evaluiert werden, welche Möglichkeiten die Digitalisierung der Lehre bietet, welche Aufgaben sich daraus für das gesamte Bildungssystem, für die Lehrenden und Lernenden ergeben, vor welchen Problemen wir Dozenten standen und stehen und welche Lösungsansätze sich ergaben. Fokus der Betrachtungen sollen dabei nicht das detaillierte Auflisten und die Erfahrungen mit einzelnen Webmeeting-Portalen oder der Einsatz von spezifischer Soft- und Hardware sein. Vielmehr ging es den Autoren darum, einen Schritt zurückzutreten und das gesamte System der Ausbildung zu betrachten.

Bei der Analyse der Vorbereitung, Durchführung und Nachbereitung der eigenen Lehrveranstaltungen kristallisierte sich bei den Autoren ein zentraler Punkt heraus: Selbstdisziplin. Das mag nun erst einmal verwunderlich klingen. Die Wahl auf diese Begrifflichkeit fiel daher, da sowohl die Umstellung der eigenen
Lehre auf digitale Formate und der damit verbundene Aufwand bzgl. Zeit und auch Kosten auf Seiten der Dozenten, als auch die konstante aktive Teilnahme der Studierenden/ Schüler auf der anderen Seite des Bildschirms abhängig sind, von der eigenen Selbstdisziplin.

Der Frage, wie Selbstdisziplin im Kontext der Ausbildung getriggert werden kann und wie sie in Zukunft gelehrt bzw. unterstützt werden muss, stellt sich der folgende Artikel.

\section{Wie Lernen Wir?}

Um einer komplexen Frage nach dem „wie lernen wir?" nachzugehen, ist die Suche nach dem Training der Selbstdisziplin und Selbstorganisation in unserem Bildungssystem ein zentraler Kernpunkt.

Während der jahrelangen schulischen Ausbildung wurden Kinder von einer Lehrkraft im Präsenzunterricht ausgebildet. Für die Kinder ist hier der direkte Kontakt, die Möglichkeit, Fragen direkt zu stellen, nonverbale Kommunikation zu lernen und auch der Vergleich mit Gleichaltrigen möglich.

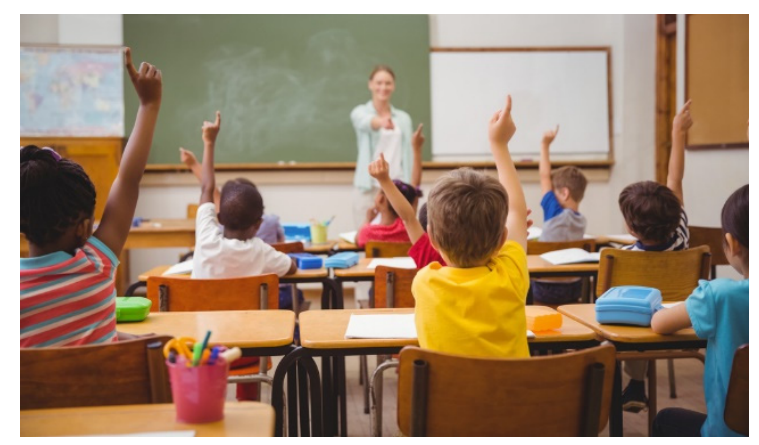

Abb. 1: Lehrer-Schüler-Kommunikation in Prösenz [1]

Dieses Konzept basiert auf dem Ansatz, dass in jungen Jahren möglichst viel Führung von außen benötigt wird, um den Kindern das „Richtig" und "Falsch“ beizubringen. Verfolgt man den schulischen Bildungsweg weiter, so wird allerdings schnell deutlich, dass sich trotz des zunehmenden Alters wenig am Führungsstil des Bildungssystems ändert. Der Fokus liegt häufig auf den zu lernenden und abzuprüfenden (prüfbaren) Inhalten und weniger auf dem "Wie" und "Warum". Im regulären Präsenzunterricht existiert eine Anwesenheitspflicht, die grundsätzlich nicht in Frage gestellt wird, 
ebenso wie klar definierte Aufgaben, die im Unterricht und als Hausaufgabe erfüllt werden müssen. Natürlich steigt der Bedarf für Selbstdisziplin mit Alter. So müssen die Schüler selbst einplanen, wann sie z.B. damit beginnen, ein Gedicht zu lernen oder ein Poster zu gestalten. Allerdings sind bis in die Abschlussjahrgänge stetig ein Lehrer bzw. die Eltern zur Stelle, um die Schüler an ihre Aufgaben während des Schuljahres zu erinnern. Und im Notfall existieren noch diverse Möglichkeiten die "Note wieder auszubügeln", wenn doch mal etwas schiefgeht.

Im Rahmen der Covid19-Pandemie wurde nun aber mehr und mehr deutlich, dass dieses Vorgehen grundsätzlich überdacht werden sollte. Nicht nur die Schüler und Eltern sind in den meisten Fällen maßlos überfordert mit der Ausbildung im digitalen Format, sondern ein ähnliches Bild zeigte sich, vor allem in den ersten Monaten, bei den Studierenden. Das „an die Hand nehmen" fehlte. Der Tag war lang, die Aufgaben schienen auf dem Blatt eigentlich überschaubar... wieso also gerade jetzt damit anfangen und nicht etwas später?

Unser Bildungssystem muss also daran ansetzen, „Lernenden“ zeitnah eigene Verantwortung zu lehren. Das "Warum" und das "Wie" spielen hierbei eine wichtige Rolle, um das Verständnis, die Notwendigkeit und die Vorteile dieser Kehrtwende in einen kausalen Zusammenhang für alle Betroffenen zu rücken.

Das "geführte Lernen" endet nun aber nicht am Schultor. Hinsichtlich des universitären Bildungssystems ist eine ähnliche Tendenz erkennbar. Vor allen in MINT-Fächern existieren in Deutschland ebenfalls mehr oder weniger feste Stundenpläne mit Lehrveranstaltungen, die den Studierenden ans Herz gelegt werden und die zum Bestehen der entsprechenden Prüfung sowie zum finalen Abschluss benötigt werden. Die Bezeichnung „obligatorisch“ wird allerdings ab dem Eintritt ins „Erwachsenenalter" scheinbar in einigen Fällen eher frei übersetzt und verstanden. Mit Prüfungen am Ende des Semesters, die in vielen Fällen nahezu $100 \%$ der Endnote ausmachen, kann man sich gut vorstellen, dass diese Prüfungsstrategie allein zur Entwicklung von Selbstdisziplin führen muss. Logische Konsequenz sollte also sein, dass die Studierenden kontinuierlich und aktiv an den Lehrveranstaltungen teilnehmen und genau wie die Dozenten - selbige vor- und nachbereiten. In der Realität wird allerdings häufig die Aufschiebe-Taktik gewählt. Begriffe wie "Bulimie-Lernen" mach(t)en hier die Runde, da während des Semesters das aktive Lernen und das Selbststudium in einigen Fällen nahe Null sind, kurz vor der Prüfung allerdings nahezu alle Hebel in Bewegung gesetzt werden, um die fehlende Sachkenntnis auszugleichen.

Ein Bildungssystem muss allerdings mehr beinhalten als Vorlesungen und das Übermitteln von fachlichen Kenntnissen. Es geht nicht nur darum, wie man die korrekte Lösung findet, sondern welchen Weg man aus welchem Grund genommen hat, um eine Lösung zu finden. Deswegen ist ein tieferes Verständnis von der Ursache von Motivation zu finden. Zentraler Ansatz muss sein, wie Motivation durch unsere Bildungssysteme entwickelt und unterstützt werden kann, um eine Strategie bzgl. Lehren und Lernen im Rahmen der Lehre, und speziell der digitalen Lehre in Zeiten der Pandemie, zu finden.

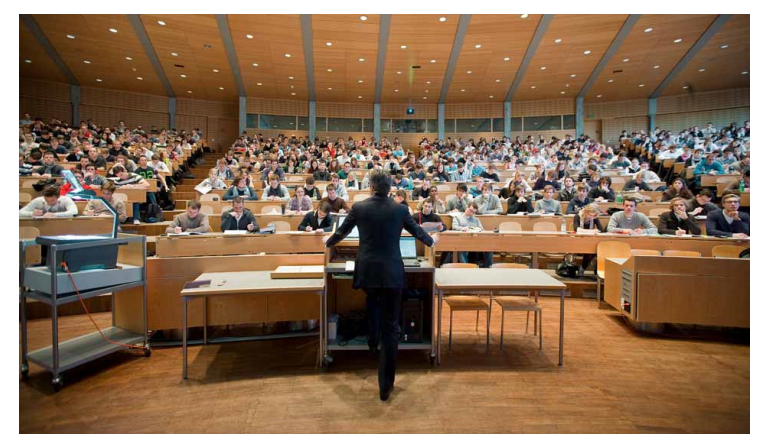

Abb. 2: Dozent-Studierenden-Kommunikation in Präsenz [2]

Nach allem Für und Wider sowie dem Ansatz, ob die Probleme der digitalen Lehrveranstaltungen vom grundsätzlichen bildungspolitischen Ansatz der Herkunftsländer der Autoren herrühren könnten, wurde offensichtlich, dass Selbstdisziplin oder disziplinarische Maßnahmen ein möglicher Schlüssel einer „erfolgreichen" Lehrveranstaltung zu sein scheinen. Mit dieser Begrifflichkeit ist hierbei nicht ein besonders guter Notenschnitt bei der abschließenden Prüfung anvisiert, sondern eine regelmäßige und interessierte Teilnahme der Auszubildenden sowie ein Verinnerlichen der The- 
matik und die Fähigkeit der Anwendung des Erlernten. Aber nicht nur auf der Seite der Lernenden muss das Thema Selbstdisziplin beleuchtet werden: ein motivierter Dozent mit frischen Ideen, eine didaktisch wertvolle Aufbereitung des Lehrinhaltes sowie eine fruchtbare Interaktion zwischen dem Dozenten und den Studierenden sind ebenfalls für ein zufriedenstellendes Ergebnis der Ausbildung nötig. Das Erfüllen der Wünsche und Bedürfnisse der Dozenten und der Studierenden sind allerdings im Rahmen einer digitalen Lehrveranstaltung noch schwerer erzielbar als beim Präsenzunterricht. Daher muss der Fokus in der "frühen Ausbildung" auf selbstinitiativem und aktivem Lernen liegen und selbiges muss durch den "Lehrkörper" unterstützt werden. Da diese Umstellung ein langer Prozess sein wird, müssen in allen Ebenen des Bildungssystems die Notwendigkeit der Selbstdisziplin, des Selbststudiums und des Hinterfragens von Fakten und Lösungsansätzen gelehrt, aufgefrischt und unterstützt werden.

\section{Verbindung zwischen Selbstdisziplin und Lehrveranstaltungen während der COVID19-Pandemie}

Betrachten wir nun die oben genannten Fragestellungen und Thesen aus Sicht der Dozenten, ergibt sich folgendes Bild: Die Mehrzahl der Dozenten, die die Lehre als Berufung und nicht nur als Beruf sehen, nehmen in Pandemiezeiten und auch vorher entsprechende Weiterbildungen wahr, setzen sich mit neuen Tools, Programmen, Ansätzen, Hardware und Software auseinander. Die COVID19-Krise stellte damit eine - wenn auch sehr hart erzwungene - Ursache und Möglichkeit für die Weiterentwicklung der Lehre dar. Zum Start des Sommersemesters 2020 mussten pragmatische Lösungen gefunden werden. Die erste Vorlesungswoche stand kurz bevor, das Land war im Lockdown. Demnach wurden seitens der Lehrer und Dozenten erstmal alle bekannten Werkzeuge verwendet und abgewandelt - immer mit Blick darauf, dass es sich hoffentlich um eine kurze Zeit handelt, in der DistanzLehre stattfinden muss. Nach einigen Tagen und Wochen in diesem Betrieb wurde allerdings absehbar, dass der Aufwand und die
Qualität der Lehre bei diesem Vorgehen gravierende Folgen für die Bildung mit sich bringen: So wurden teilweise einfach nur die Folien im pdf-Format ins Netz gestellt. Andere Ansätze wie "vertonte Präsentationen mit PowerPoint" trieben die Lehrenden (die Autoren eingeschlossen) an den Rand des Wahnsinns, da die Qualität der Tonspur nicht zufriedenstellend war und man bei einem kleinen Versprecher oder einem kurzen Stocken, die Folie lieber nochmal von Anfang an eingesprochen hat. Nun kann man sich selbst als Außenstehender gut vorstellen, dass beim dritten, vierten, fünften Anlauf an eine Folie, die Motivation rapide abnimmt. Selbstdisziplin bei den Lehrenden war auch hier zwingend nötig! Die Zeitinvestition zur Vorbereitung nahm rasant zu und es musste nach Lösungen gesucht werden, die Qualität der Lehre wieder zu verbessern und den eigenen wöchentlichen zeitlichen Aufwand wieder zumindest teilweise zu reduzieren. Das wiederum bedeutete: Selbstdisziplin, Selbstinitiative und Selbststudium seitens der Lehrenden: Neue Tools, neue didaktische Ansätze, neue Hardware waren gefragt! Nach mehr als einem Jahr Pandemie sind nun diverse Werkzeuge bekannt, die sich spezifisch für die digitale Lehre eignen und von denen einige mit großer Wahrscheinlichkeit auch bei hybriden Konzepten oder dem von den meisten Dozenten ersehnten Präsenzunterricht zukünftig eingesetzt werden.

Viele solcher Ideen werden weltweit und auch an der TU Dresden umgesetzt. Die Lessons Learned-Veranstaltungen im Herbst 2020 und Frühjahr 2021 zeigten das große Engagement der Dozenten: Die Erfahrungen mit neuen Programmen wie bspw. dem vorlesungsspezifischen Paella-Player [3] wurden in diesem Rahmen geteilt. Ziel dieses Werkzeugs ist, dass sich die Vorlesungserfahrung für den Dozenten sowie die Studenten durch ein vereinfachtes Navigieren zwischen den Folien und dem Video verbessert und einfache Möglichkeiten existieren, um den Bildschirm zu individualisieren. Weitere Ansätze wie die Berichte über den Einsatz von Greenscreen-Technik [4] lieferten wichtige Erkenntnisse bzgl. der Vor- und Nachteile. Mit Hilfe dieser Technologie kann sich der Dozent in den Vortrag selbst integrieren und damit besser mit seinen Lehrinhalten intera- 
gieren. Solche Ideen trieben die Weiterentwicklung von Bildung trotz schwieriger Randbedingungen voran und sind Vorbild für den Prozess "Erfindung durch Bedarf".

Da die Autoren dieses Artikels in den bisherigen "Corona-Semestern" lediglich im Bereich der Vorlesungen agierten, beschränken sich auch die Erfahrungen hierauf. Nach einer Vielzahl an Überlegungen, getesteten Tools und neuen Ansätzen, entschieden sich die Autoren für den Einsatz der Webmeeting-Plattform Zoom, u.a. aufgrund der Stabilität der Plattform und der Möglichkeit der Aufnahme der Lehrveranstaltung. Dies stellte einen entscheidenden Vorteil im Vergleich zu bspw. BigBlueButton dar, da somit auch die Studierenden Zugang zu den Inhalten hatten, die aufgrund des Lockdowns, Kinderbetreuung etc. nicht an einer synchronen Veranstaltung teilnehmen konnten. Das Einbinden von Videos für eine bessere Erklärung der Vorgänge in den betrachteten Systemen sowie die Möglichkeit des Einsatzes von Umfrage-Tools wie Invote, Kahoot! usw. sind zusätzlich für die "synchron"-Teilnehmer nutzbar und einfacher und stabiler einzubinden als bei anderen Plattformen.

Da Vorlesungen allerdings nur ein Teil des Lehrangebots an der TU Dresden darstellen, waren und sind auch neue Ideen für die Durchführung Praktika ohne Präsenz gefragt. Laborpraktika von der physikalischen Chemie wurden bspw. durch Ideen wie Lab@Home [5] durchgeführt, damit den Studierenden ein tieferes Verständnis von komplexen Sachverhalten nähergebracht werden konnte. Die bisher gemeinsam im PC-Pool durchgeführten Aufgaben wurden nun in die Bearbeitung zu Hause (Lab@Home) überführt, wobei eine direkte Betreuung seitens des Lehrstuhls online eingerichtet wurde. Ein weiteres hervorragendes Beispiel an der TU Dresden stellt eine Veranstaltung im Rahmen eines physikalischen Praktikums dar [6], wobei Maschinen virtuell dargestellt wurden und die Studierenden selbige selbstständig am heimischen Bildschirm aus verschiedenen Winkeln betrachten konnten, was zum besseren Verständnis der Funktionsweise beiträgt. Natürlich ist dieses Vorgehen nicht vergleichbar mit dem tatsächlichen Berühren und Ausprobieren von Komponenten und Maschinen, aber es stellt einen logischen
Zwischenschritt zwischen der gewünschten haptischen Erfahrung und einem Bild als pdf dar.

Die benannten Beispiele zeigen nur einen Bruchteil der an der TU Dresden eingesetzten Tools und Ansätze. Grundsätzlich muss folgendes auf der Seite der Dozenten festgehalten werden:

Der Wille ist da, die Möglichkeiten nahezu unerschöpflich, die Zeit jedoch recht begrenzt.

Nach Gesprächen mit Dozenten und Übungsleitern während der ersten beiden "Corona-Semester" wurde deutlich, dass in den meisten Fällen die Live-Zuhörerschaft rapide abgenommen hat. Im Vergleich zu den im Bildungsportal OPAL eingetragenen Teilnehmern, gratulierte man sich teilweise intern schon zu $10 \%$ dieser Menge, die an den wöchentlichen Veranstaltungen teilnehmen. Bei all dem Aufwand, der in die Vorbereitung der digitalen Lehre gesteckt wird, gepaart mit der Tatsache, dass die Kameras und Mikrofone der anderen Seite der Bildschirme während der Vorlesung zumeist dunkel und stumm sind, ist der Frustrationsgrad auch auf der Seite der Lehrenden fortschreitend. Bzgl. der reduzierten Teilnahme an der synchronen Veranstaltung scheint ein wichtiger Punkt im Vordergrund zu stehen: Wird die live gehaltene Vorlesung anschließend digital zur Verfügung gestellt (inkl. Ton und Bild) oder nicht.

Fragen, die sich den Autoren hierbei stellen sind die folgenden: Genügt es den Studierenden, vertonte Lehrveranstaltungen anzuhören und zu sehen? Gefällt den Studierenden genau dieser offline-Lehrbetrieb sogar besser als der Präsenzunterricht?

Nach Rücksprachen mit einzelnen Studierenden kristallisierte sich die freie zeitliche Einteilung des "Anhörens" der Lehrinhalte als maßgeblicher Grund heraus. Sind es demnach evtl. nur (bzw. schwerpunktmäßig) die Dozenten, die sich die Lehre im Hörsaal zurückwünschen? Und: Wie kann man die aktive Teilnahme der Studierenden an der Online-Lehre erhöhen, um eine aktive Lehrerfahrung bei den Studierenden hervorzurufen und das „BulimieLernen" zu reduzieren?

Augenscheinlich wird eine Vielzahl an Methoden der digitalen Lehre an der TU Dresden angeboten. Selbige können aber das aktive und 
gemeinsame Lernen der Studierenden und das Begleiten durch den Dozenten im Rahmen der Lehrveranstaltungen und auch darüber hinaus nicht ersetzen. Das regelmäßige Arbeiten seitens der Studierenden muss angestrebt werden. Wissen kann nicht sinnvoll und langfristig innerhalb weniger Tage erlangt werden. Das heißt, es müssen Wege gefunden werden, wie der Prozess des Lernens über das gesamte Semester hinweg gefördert und auch gefordert werden kann.

Betrachtet man nun nochmal den eigenen Bildungsweg, die eigene Schulzeit, so gab es prinzipiell zwei Möglichkeiten, warum eine Aufgabe durchgeführt wurde: Entweder war das Bewusstsein vorhanden, dass es nötig ist, den einen oder anderen Lehrinhalt zu verstehen und anwenden zu können - was beinhaltet, dass der kausale Zusammenhang bis hin zum eigenen Nutzen des Erlernten bekannt ist. Oder es bestand eine klare Forderung inkl. einer direkt damit verbundenen Konsequenz/ Sanktion, falls die Aufgabe nicht erfüllt wurde.

Im ersten - natürlich angestrebten Fall - ist es die Aufgabe des Lehrkörpers darzustellen, warum eine Aufgabe durchgeführt werden sollte und was das Ziel der Lehrveranstaltung und der abschließenden Prüfung ist. Allerdings muss hier hervorgehoben werden, dass es sich bei den Teilnehmern einer universitären Lehrveranstaltung um junge Erwachsene handelt, die ihren Studiengang selbst gewählt haben und bestrebt sein sollten - also intrinsisch motiviert - nicht nur an den Veranstaltungen teilzunehmen, sondern aktiv daran teilzunehmen. Hier müsste im Grunde zu einem weitaus früheren Zeitpunkt, am besten im Kindesalter, der kausale Zusammenhang zwischen Lernen, eigenem Einsatz von Zeit und Ressourcen und eigener (beruflicher) Zielstellung gelegt werden.

Kommt dieser Weg nicht an, so ist natürlich auch der zweite genannte Weg eine Möglichkeit: Konsequenzen ziehen in Form von "Zwischenprüfungen“. Selbige können nun sein, dass das Teilnehmen an einer Lehrveranstaltung als verpflichtend definiert und geprüft wird. Somit wird den Studierenden, die mehr Struktur und Führung benötigen, eine entsprechende Hilfestellung angeboten und alle Teilnehmer könn(t)en regelmäßig die Inhalte der
Lehrveranstaltungen diskutieren. Ein weiterer Schritt zum regelmäßigen Lernen sind - nach dem schulischen Vorbild - Hausaufgaben, oder Quizze während der Lehrveranstaltung, die ein Teil der Modulnote ausmachen. Diese Ansätze werden im universitären System in Deutschland eher wenig gelebt. In anderen Länder, beispielsweise im Heimatland einer der Autoren dieses Artikels, den USA, werden diese Ideen regelmäßig umgesetzt.

Natürlich gibt es bei den benannten Ansätzen stets Vor- und Nachteile, die vorsichtig betrachtet werden müssen. Allerdings bedarf eine Situation wie wir sie während der COVID19-Pandemie vorfinden so vieler Ideen, Vorschläge und alternativer Ansätze wie möglich.

Vor allem im Hinblick auf das Thema Selbstdisziplin ist der zweite, in diesem Abschnitt genannte Ansatz aus Sicht der Autoren langfristig der „Falsche“. Man bindet so die Studierenden zwar an das eigene Fach, allerdings muss im Bildungssystem grundsätzlich wieder klargemacht werden, dass es sich bei der Ausbildung um ein Privileg handelt, das nicht jedem Menschen ermöglicht wird, und der eigene Drang nach Fort- und Weiterbildung und beruflichem Erfolg seitens der Dozenten unterstützt wird und es keine Strafe ist, an Lehrveranstaltungen teilzunehmen.

\section{Vorschläge und Diskussion: Was ist sinnvoll und nützlich?}

In Gesprächen mit einzelnen Studierenden sowie auch schriftlichen Rückmeldungen wurde deutlich, dass "einfache pdfs ohne Kommentare wenig hilfreich" sind und bei den Studierenden als "unmotiviert" seitens der Lehrperson betrachtet werden. Vertonte Varianten oder aufgenommene Lehrveranstaltungen sind hingegen hochgeschätzt. Die Qualität der Aufnahme, die Anzahl der eingebauten Gimmicks, auf die wir Lehrpersonen so stolz sind (aufgrund der Menge an Zeit und des schönen didaktischen Ansatzes), scheint hierbei eher weniger wichtig zu sein. Hauptsache, es ist „etwas da, was erklärend hinzu kommt".

Auf die Nachfrage, warum die Kameras seitens der Studierenden ausgeschaltet werden, kam eine für die Autoren verblüffende, aber den- 
noch recht logische Antwort. "Wir sehen Sie ja und haben damit Kontakt. Dass das für Sie komisch ist, hätten wir nicht gedacht". Dem Wunsch, die Kameras einzuschalten, um besser zu interagieren, kommen einzelne Teilnehmer nach, allerdings gilt auch hier, dass jede Stunde daran erneut erinnert werden muss. Zielstellung für die digitale Lehre sollte allerdings eher die gemeinsame Lehrveranstaltung sein - sowohl inhaltlich, als auch visuell und akustisch (siehe Abb. 3).

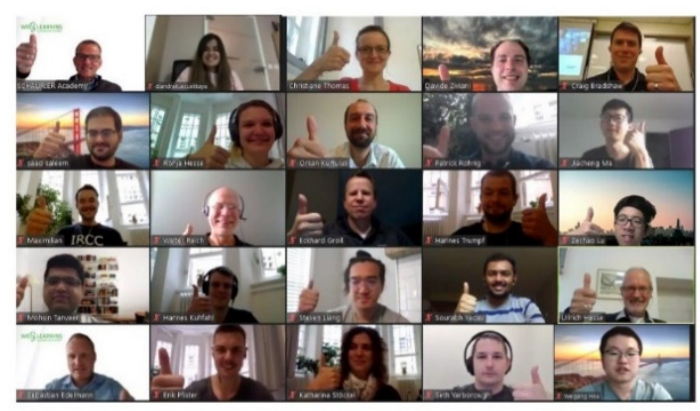

Abb. 3: Teilnehmer International Refrigeration and Compressor Course, TU Dresden, 2020

Im Selbsttest, wie vertonte PowerPoint-Lehrveranstaltungen „zu verarbeiten“ sind, die nicht live besucht werden, kamen die Autoren zu dem Schluss, dass 90 Minuten konzentriertes Zuhören nicht möglich ist - die Gedanken driften $a b$, wenn keine Fragen gestellt werden, die eine Antwort erfordern und kein dringend benötigter Medienwechsel stattfindet. Denn das Medium - trotz der liebevoll aufbereiteten Videos, Animationen, eingesetzten Tablets, usw. - ist und bleibt das mp4/ppsx-Format. Auf Nachfrage bei den Studierenden wird genannt, dass die Lehrveranstaltungen "schneller abgespielt" werden und bei "interessanten Dingen" die Geschwindigkeit wieder angepasst wird. Nachvollziehbar? Durchaus. Zielführend im Hinblick auf die Tiefe des verstandenen Lehrstoffes? Wohl kaum.

Eine Alternative zu 90-minütigen Blockvorlesung sind kürzere Mitschnitte. Versucht wurden zum einen 60-minütige Vorlesungen mit anschließender Diskussions- und Fragemöglichkeit. Das Ergebnis war, dass wenige bis keine Fragen gestellt wurden und die Veranstaltung wenige Minuten später beendet werden konnte. Lehrblöcke von $15 \mathrm{~min}$ sind durchaus möglich, um dem Geschehen konzentriert zu folgen. Die Frage nach der Reduktion des Lehrinhaltes steht jedoch im Raum. Selbst bei drei 15 min-Lehrveranstaltung, verbleiben 45 min des früheren Lehrstoffes. Einfache Möglichkeit: Selbststudium! Das führt uns erneut zum Punkt der Selbstdisziplin. Wenn in Pandemiezeiten "Hausaufgaben“ gegeben werden, die wir liebevoll "Selbststudium" in der Erwachsenenbildung nennen: Wie werden diese erfüllt? Ernüchternde Resultate sind in einigen Fällen vermutlich vorprogrammiert. Die Frage ist nur, warum war das in der Schule möglich und ist heute so schwierig? Ein unbehelligtes Semester und eine finale Prüfung scheinen hier das Problem zu sein. Kurze Tests, am besten unangekündigt, scheinen eine mögliche Lösung. Das Problem scheint dabei die DPO zu sein: Eine Prüfung, keine geforderte aktive Teilnahme an der Lehrveranstaltung, Ende. Die Studierenden sind mit Ihrer (vorhandenen oder nicht vorhandenen) Selbstdisziplin alleingelassen. Ist das hilfreich? Für eine Seite, beide Seiten oder am Ende doch für gar keine?

Vielleicht sind Ansätze, wie in Abb. 4 aufgeführt, sinnvoll, für einen Start in die Vorlesung.

\section{Sticker für Meeting-Ziele}

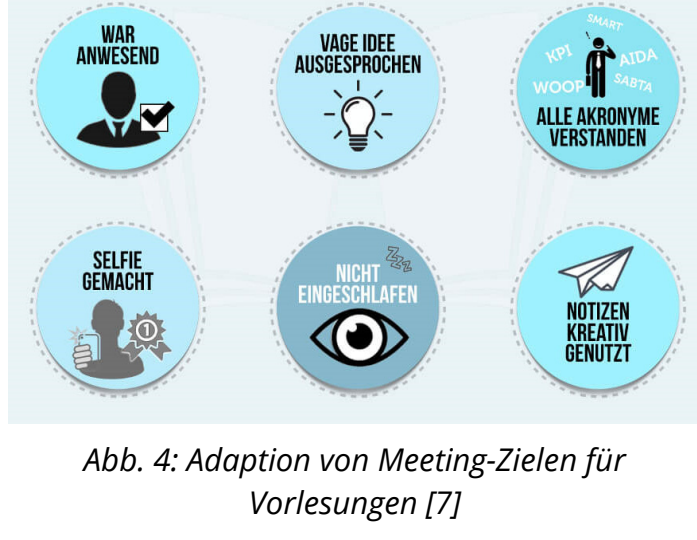

Denken wir an die Vielzahl an Online-Meetings, die Tag für Tag stattfinden und wie schnell man "nebenher mal schnell" noch eine E-Mail schreibt. Selbstdisziplin ist auch hier erneut in den Vordergrund zu rücken. Einen neuen Ansatz, der im kommenden Sommersemester ganz oben auf der "trial and error"-Liste steht, ist der Vorschlag, sich zwei zu erfüllende Ziele 
für die jeweilige Lehrveranstaltung auszusuchen, wie bspw. „Kamera an“ und „mindestens eine Frage in den Chat gestellt". Die Ziele aus Abb. 4 sind hierbei selbstverständlich plakativ zu sehen und sollten entsprechend angepasst werden.

Ziel der Lehre war für uns immer ein begleitendes Lehren und Lernen. Die Kommunikation ist mit schwarzen Bildschirmen im Zoom-Call gleich null. Disziplinarische Maßnahmen, und sei es nur ein kleines Quizz, offiziell (zumindest in den Modulen der Autoren) nicht möglich, es sei denn, man geht den Weg einer Änderung der Prüfungsordnung. Das Einbinden kleiner Kniffe wie Multiple Choice Fragen in die Vorlesung werden von den Teilnehmern (die, die synchron teilnehmen) wertgeschätzt und absolviert. Diskutieren möchten dann aber relativ wenige über die gelieferten Antworten. Vielleicht ist mit gemeinschaftlich gesteckten Zielen (Kamera an, Frage gestellt, ...) und einer noch größeren Offenheit der Lehrperson gegenüber den Studierenden bzgl. des (nicht-inhaltlichen) Ablaufs der Veranstaltung eine Verbesserung der digitalen Lehre möglich. Die Praxis zeigt aber auch hier, dass von einigen Teilnehmern die Bitten erfüllt werden, aber sich dann doch das gewohnte Bild der "schwarzen Bildschirme“ über das Semester wiedereinstellt.

Bei den internen Diskussionen zur Lehre zwischen den Autoren zum Thema Selbstdisziplin wurde stets auch die Verbindung zum eigenen "Zeitmanagement" geschlagen. Jeder von uns hat hier seine Stärken und Schwächen. Einen inneren Schweinehund haben wir alle. Die Frage ist nur, wie können wir die Studierenden (und uns selbst) besser lehren, mit diesem umzugehen?

Der Vorschlag der Autoren ist hier evtl. für einige zu strikt, soll aber genau deshalb zur Diskussion gestellt werden:

Ein obligatorischer Kurs zu Selbstdisziplin, ein „How-To" (Online-)Mitarbeiten und Zeitmanagement für alle Studierenden zum Studienbeginn bzw. nächsten Semesterstart mit anschließender Prüfung an Fallbeispielen. Und um einen Schritt weiter zu gehen: die Integration dieser "Schulungen" auch in den schulischen Alltag, damit die Ursachen und Wege zu einem frühen Zeitpunkt geklärt werden können.

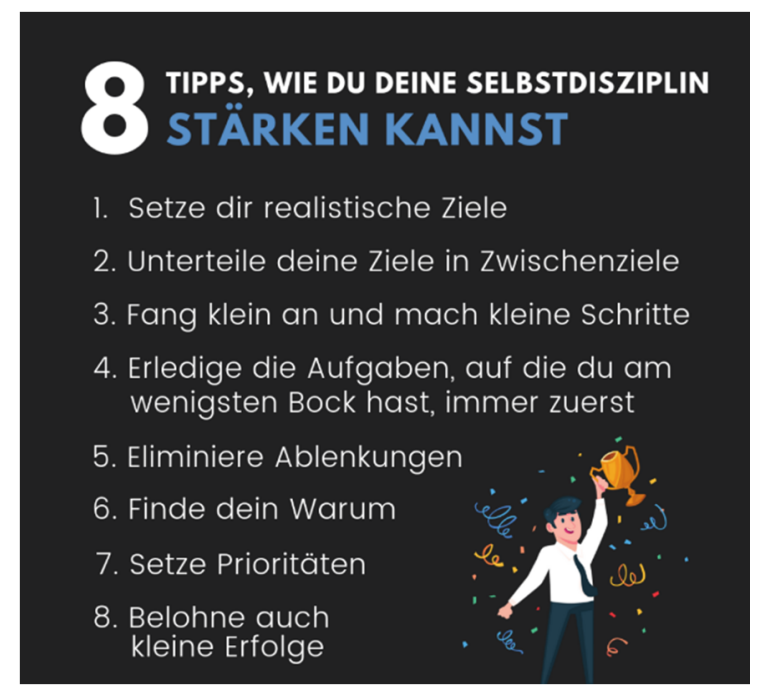

Abb. 5: Selbstdisziplin [8]

Der Mehrwert einer solchen Veranstaltung wird seitens der Autoren als sehr hoch eingeschätzt - nicht nur für das Studium, sondern auch für das spätere Berufs- und Privatleben.

\section{Zusammenfassung}

Der vorliegende Artikel wurde nach einem Jahr voller Wissensgewinn hinsichtlich neuer Softund Hardware und den daraus resultierenden Erfahrungen geschrieben. Neue Kniffe, Videoschnitt-Programme, Tablets, Online-MeetingTools, Beleuchtung, Vertonung, vorteilhafte Videoeinstellungen, Dos and Don'ts wurden kennengelernt und getestet - neben den alltäglichen Aufgaben. All das war nur möglich, mit einer gehörigen Portion an Enthusiasmus und Selbstdisziplin, die eine Vielzahl an Dozenten und Studierenden auch besitzen. Beide "Seiten" benötigen $a b$ und an einen Anstoß für eine gute und zufriedenstellende Lehrveranstaltung. Und eine Solche ist aus Sicht der Autoren nur möglich, wenn der Dozent nicht ins große "Off" spricht, sondern der reguläre Betrieb und eine aktive Teilnahme so gut wie möglich in das digitale Format überführt wird.

Wir hoffen, dass diese Stellungnahme keinen zu negativen Tenor aufweist. Der Unterschied zwischen Wunsch und Realität musste im vergangenen Jahr mehrmals hinterfragt werden. Nicht jedes Tool, das die Dozenten begeistert, 
ruft diesen Enthusiasmus bei den Studierenden hervor bzw. ist fraglich, ob der Aufwand und der Nutzen für die Teilnehmer sichtbar wird und größere Erfolge erzielt. Dieser Artikel teilt damit vermutlich mehr die eigenen „Lessons Learned" der Autoren: vom Kennenlernen der eigenen Frustrationsschwellen, unglaublichen und nicht erwarteten Aha-Erlebnissen über die Nutzung völlig neuer Ansätze bis zum Erblicken des "Lichts am Ende des Tunnels".

Als abschließende Worte und Verbindung zur These und zum Aufruf nach mehr Selbstdisziplin und Selbstorganisation, sei allen Teilnehmern einer Lehrveranstaltung - Dozent oder Studierender - nochmal die Bedeutung des Wortes "studieren“ ins Gedächtnis gerufen. Sowohl Lehrende als auch Lernende sind, aus Sicht der Autoren, stets als Studierende (studens - lateinisch) zu betrachten, denn sie sollten nach Wissen und Weiterbildung gemäß der Wortdefinition "streben" und sich um den eigenen Fortschritt und den der anderen „bemühen".

\section{Literatur}

[1] HEROLÉ, „Herolé Ratgeber,“ [Online]. Available: https://www.herole.de/blog/wp-

content/uploads/schueler-im-klassenzimmer.jpg. [Zugriff am 0103 2021].

[2] Forschung \& Lehre, „Forschung \& Lehre," [Online]. Available: $\quad$ https://www.forschung-undlehre.de/fileadmin/user_upload/Rubriken/Politik/20 18/6-18/Professor_c_mauritiusimages_Keyston_Gaetan-Bally_06039086.jpg. [Zugriff am 0103 2021].

[3] Stelzer, R. (2021). „Online-Vorlesungen mit dem Paella-Player, "Lessons Learned II - Spin Offs digitaler Lehrerfahrungen", TU-Dresden, März 2021.

[4] Beitelschmidt, M., Bernstein, D., Bieber, J. und Schuster, M. (2021). „Produktion von Vorlesungsvideos mit Greenscreen-Technik, "Lessons Learned II - Spin Offs digitaler Lehrerfahrungen“, TU-Dresden, März 2021.

[5] Röder, F., und Schwierz, R. (2021). „Virtuelles Physikalisches Praktikum an der TU Dresden für Studierende der Physik, "Lessons Learned II - Spin Offs digitaler Lehrerfahrungen", TU-Dresden, März 2021.

[6] Kampmann, S., Bodesheim, D., Croy, A., Gutierrez, R., Dianat, A. und Cuniberti, G. (2021). "Virtuelle PC Pools für Computerüpraktika in der materialwissenschaft, "Lessons Learned II - Spin Offs digitaler Lehrerfahrungen“, TU-Dresden, März 2021.

[7] karrierebibel, „karrierebibel,“ [Online]. Available: https://karrierebibel.de/wpcontent/uploads/2017/04/Meeting-StickerMeetingitis.jpg. [Zugriff am 0203 2020].
[8] einfachtaeglich, "einfachtaeglich," [Online]. Available: https://einfachtaeglich.de/wpcontent/uploads/2019/07/8-Tipps-wie-du-deineselbstdisziplin-st\%C3\%A4rken-und-trainierenkannst-1024x1024.png. [Zugriff am 0203 2020]. 